\title{
Aprender a pensar históricamente. Retos para la historia en el siglo XXI
}

\section{Resumen}

El objetivo de este artículo es reflexionar sobre la construcción del conocimiento histórico y su influencia en los procesos de enseñanza y aprendizaje a través del dossier "Historia y Pensamiento Histórico". Este trabajo incide en la necesidad de adentrarse en la epistemología de la disciplina para conectar con el significado de la comprensión histórica. Contrariamente a la creencia popular, saber historia no equivale a la acumulación memorística de hechos, conceptos o fechas del pasado. La comprensión histórica implica unos complejos procesos de pensamiento que permitan interpretar esos contenidos (conceptos de primer orden), a través de estrategias propias de la disciplina (conceptos estratégicos o de segundo orden). Para adquirir estas habilidades es necesario la participación de los alumnos en la práctica del historiador. Este artículo realiza un repaso de la literatura temática sobre el pensamiento histórico para buscar los elementos comunes que permitan introducir estos procesos de aprendizaje en las aulas. Para desarrollar esta cuestión es necesario que los docentes posean tanto un conocimiento teórico sólido sobre la epistemología de la disciplina (qué es la historia), como del aprendizaje y búsqueda de marcadores de progresión cognitiva en los alumnos (qué significa la comprensión histórica y pensar históricamente)

Palabras clave: Pensamiento histórico; Epistemología; Enseñanza de la historia.

\section{Cosme Jesús Gómez Carrasco}

Licenciado en Humanidades y Doctor en Historia por la Universidad de

Castilla-La Mancha. Profesor Contratado Doctor de Didáctica de las Ciencias Sociales de la Universidad de Murcia. cjgomez@um.es

\section{Jorge Ortuño Molina}

Licenciado y doctor en Historia por la Universidad de Murcia. Profesor de Didáctica de las Ciencias Sociales en la Universidad de Murcia, donde ocupa plaza de profesor Contratado Doctor. jortunom@um.es

\section{Sebastián Molina Puche}

Licenciado en Geografía e Historia y Doctor en Historia por la Universidad de Murcia. Profesor titular de Didáctica de las Ciencias Sociales de la

Universidad de Murcia. smolina@um.es

\section{Para citar este artículo:}

GÓMEZ, Cosme Jesús; ORTUÑO, Jorge; MOLINA, Sebastián. Aprender a pensar históricamente. Retos para la historia en el siglo XXI. Revista Tempo e Argumento, Florianópolis, v. 6, n.11, p. 05-27, jan./abr. 2014.

\section{DOI: $10.5965 / 2175180306112014005$}

http://dx.doi.org/10.5965/2175180306112014005

\footnotetext{
1 Este trabajo es resultado del proyecto "La evaluación de las competencias básicas en Educación Secundaria Obligatoria desde las ciencias sociales" (EDU2012-37909-C03-03), subvencionado por el Ministerio de Economía y Competitividad y del proyecto "Familia, curso de vida y reproducción social en la España centro-meridional, 1700-1860", referencia HAR2010-21325-C05-03 financiado por el Ministerio de Ciencia e Innovación.
} 


\section{Learning to think historically. Challenges for history in the 21st century}

\begin{abstract}
The aim of this paper is to reflect how historical knowledge is constructed and taught, and to present the different contributions made by different authors in this special issue about "History and Historical Thinking". This paper highlights the need to delve into the epistemology of the discipline to connect with the meaning of historical understanding. On the contrary of popular belief, knowing history is not an accumulation of facts, concepts or dates of the past. The historical understanding involves complex processes of thinking about how to interpret that content (concepts of first order) through discipline strategies (strategic or second order concepts). In order to acquire these skills students are required to act and think like a historian. This article reviews relevant literature about historical thinking to find common elements to introduce these learning processes in classrooms. To develop this question it is necessary that teachers possess both a solid theoretical understanding of the epistemology of the discipline (what history is), and the pursuit of learning and cognitive markers of progression in students (to know whether historical understanding and thinking historically is achieved or not).
\end{abstract}

Keywords: Historical Thinking; Epistemology; History Education.

\section{Aprender a pensar historicamente. Desafios para a história no século XXI}

\section{Resumo}

O objetivo deste artigo é refletir sobre a construção do conhecimento histórico e a sua influência nos processos de ensino e aprendizagem através do dossiê "História e pensamento histórico". Este trabalho enfatiza a necessidade de investigar a epistemologia da disciplina para conectar com o significado da compreensão histórica. Contrariamente à crença popular, saber história não equivale à acumulação mnemônica de fatos, conceitos ou datas do passado. A compreensão histórica implica alguns processos complexos de pensamento que permitam interpretar esses conteúdos (conceitos de primeira ordem), por meio de estratégias próprias da disciplina (conceitos estratégicos ou de segunda ordem). Para adquirir estas habilidades, é necessária a participação dos alunos na prática do historiador. Este artigo passa em revista a literatura temática sobre o pensamento histórico para buscar os elementos comuns que permitam introduzir estes processos de aprendizagem nas aulas. Para desenvolver esta questão é necessário que os docentes possuam tanto um conhecimento teórico sólido sobre a epistemologia da disciplina (o que é a história), como da aprendizagem e busca de marcadores de progressão cognitiva nos alunos (o que significa a compreensão histórica e pensar historicamente).

Palavras-chave: Pensamento histórico; Epistemologia; Ensino da história. 
Casi dos siglos de historia como disciplina académica permiten aportar elementos interesantes para hacer de esta ciencia un saber imprescindible para las sociedades del siglo XXI. Sin duda, uno de los grandes retos de la historia es tanto la capacidad de generar conocimiento e identificar los procesos históricos como los de transmitirlos y conseguir que la sociedad perciba el peso de dichos procesos a la hora de orientarse y adoptar decisiones en nuestra vida diaria. Es esta faceta la que aquí nos interesa y por la que abogamos justificando su presencia con el aval de parte de la historiografía fundamentalmente alemana- que ha incidido en la función social de la historia, dando pie a la "historia social crítica" que se caracteriza por incidir en la necesidad de la historia aplicada al servicio de la sociedad. Esta corriente historiográfica puede rastrearse desde finales del siglo XIX, sin embargo ha sido la segunda mitad del siglo XX la que ha visto cómo historiadores de la altura de Kocka, Wehler, Hans, Wolfgang Mommsen... o filósofos de la historia como Jörn Rüsen han apostado por hacer una historia "emancipadora", que ayude a los ciudadanos "a derribar la niebla de las leyendas arraigadas por medio de la crítica ideológica y deshacer los errores típicos, poner claramente de relieve las consecuencias que sufrieron los afectados o los costes sociales de las decisiones que se tomaron. Y con ello, aumentar las posibilidades de orientarnos racionalmente en nuestra vida práctica, al incluirla en un horizonte de experiencias históricas cuidadosamente comprobadas" (WEHLER, 1988; citado en MILLÁN, 2002, p. 14). El deseo de la historiografía social crítica, entendiendo su origen en el papel y la responsabilidad de la sociedad alemana en los grandes conflictos bélicos que sufrió el mundo en el siglo XX, así como su actual papel de eje clave en el capitalismo actual, se centra en una historia que permita explicar las actuaciones propias en un pasado reciente con un propósito crítico y con el declarado objetivo de influir en la opinión pública ciudadana. Uno de los conceptos historiográficos más interesantes de esta corriente ha sido el de conciencia histórica que se traduce en la valoración y apreciación que las sociedades tienen de su pasado y que condiciona su actuación presente (RÜSSEN 2005; CATAÑO, 2010). Auspiciado en parte por este paraguas historiográfico se está planteando la necesidad de una enseñanza de la historia que vaya más allá del aprendizaje de contenidos históricos adoptados como productos finitos y cerrados que no exigen en 

la metodología docente que intenta, precisamente, acercar al alumnado a la historia en su totalidad. Este reto está excediendo el ámbito académico y la formación de futuros historiadores en las universidades para ser adoptado por los enfoques didácticos de la historia en etapas educativas iniciales como la Educación Primaria y Secundaria y, por tanto generalizar, los beneficios que una formación histórica tienen para el común de la sociedad.

La dificultad de acercarse a la historia y a su enseñanza radica en que, en contra de la opinión generalizada en muchas aulas sobre que la historia es lo que ocurrió, la historia es un conocimiento que posee unas particularidades que exigen para su enseñanza la comprensión de su propia formulación. Esta última característica es la que se ha venido obviando reiteradamente por las dificultades cognitivas, principal argumentación en contra de la misma, que para etapas preuniversitarias requiere del alumnado. Sin embargo, sin el adecuado conocimiento epistemológico por parte de los enseñantes ni del alumnado la historia tiende a caer en conocimientos -datos- escasamente relevantes para una sociedad demandante de técnicos e ingenieros. Eso en la mayoría de los casos, porque los más extremos y preocupantes se dan en el uso de los acontecimientos históricos distorsionados -descontextualizados- para la justificación de acciones presentes. La disciplina histórica nos permite el acercamiento a hechos del pasado de los cuales no podemos tener conocimiento ya que, y valga la redundancia, pasaron hace tiempo. Es decir, pretendemos obtener conocimiento de algo que no podemos reproducir en sus condiciones de génesis ni hemos sido testigos directos de los mismos. Solo tenemos noticias de tales hechos por trazos de información incompleta y generadas con una intencionalidad que es necesaria contextualizar e identificar con precisión para evitar varios de los grandes problemas achacados a la disciplina histórica: la subjetividad y la inexactitud. Ante la realidad de que historia -el producto de la investigación realizada por los investigadores- y el pasado -actuaciones humanas que tuvieron lugar en tiempos ya pretéritos- no son lo mismo, y que lo que conocemos es un discurso narrativo 

noventa, debilitó la idea de la historia como un conocimiento veraz, destrozando la idea de un proceso unitario y de evolución (VATTIMO, 1989). Sin embargo, ayudó a reforzar la idea de que la historia es una narración y es un hecho que no podemos olvidar en la enseñanza de la historia. A pesar de que historia es interpretación, y de ahí la disparidad de enfoques sobre un mismo acontecimiento, afirmaba Stone (1991) que la narración se ha de asentar sobre un método riguroso que reduce el subjetivismo y que permite crear un conocimiento firme y aceptado del pasado. En esta misma línea Rüsen defiende que la historia es cambiante porque depende de la necesidad de respuestas y orientación que demandamos día a día en nuestras sociedades, aunque la historia es mucho más compleja que la mera satisfacción de las necesidades de orientación y justificación del presente por parte de los ciudadanos-historiadores. El conocimiento histórico satisface nuestra realidad diaria, pero genera un plus independiente a lo demandado. Es precisamente esa plusvalía la que justifica la historia como una ciencia más allá del relativismo social y que le otorga valor no tanto por el contenido que genera sino por el cómo lo genera (MEGILL, 1994).

Es decir, la enseñanza de la historia exige del conocimiento de la disciplina, de su epistemología, que trasladado al vocabulario educativo exige y demanda en los currículos educativos la presencia de contenidos sustantivos o contenidos de primer orden, que intentan responder a las preguntas ¿qué? ¿quién? ¿cuándo? y ¿dónde? Estamos hablando de contenidos que hacen referencia tanto a conocimientos de conceptos o principios, como a fechas y acontecimientos históricos concretos. Pero son igualmente imprescindibles los contenidos estratégicos o de segundo orden. Éstos se definen por la posesión o despliegue de diferentes estrategias, capacidades o competencias para responder a cuestiones históricas y entender de una forma más compleja el pasado. Este último tipo de conocimientos históricos están relacionados con habilidades propias del historiador que se concretan en: la búsqueda, selección y tratamiento de fuentes 
Como ya hemos indicado, tradicionalmente se ha producido una especie de dicotomía en cuanto a la "adscripción" de este tipo de contenidos: mientras que los contenidos de primer orden se convertían en el principal objeto en el que se centraba la enseñanza de la historia, los contenidos estratégicos o de segundo orden quedaban reservados casi en exclusiva a las tareas propias de la investigación en historia, dándose así una nueva muestra de la diferenciación jerárquica existente entre la enseñanza y la investigación (MOLINA, RODRÍGUEZ y SÁNCHEZ, 2013). Sin embargo, en los últimos años se ha puesto énfasis en la conveniencia de que la enseñanza de la historia tienda a la construcción del pensamiento histórico, lo que supone aprender habilidades en la interpretación del pasado, más allá de un conocimiento conceptual o memorístico (ASHBY, LEE, 1987; SEIXAS, 1996; RÜSEN, 2005; LEE, 2005b; PECK, SEIXAS, 2008; LÉVESQUE, 2008; BARCA, 2011; CARRETERO, LÓPEZ, 2009; CARRETERO, 2011; LÉVESQUE, 2011; LÓPEZ FACAL et al, 2011; SÁIZ, 2013; SEIXAS, 2010 y 2011). El reto, por tanto, es conseguir organizar una enseñanza de la historia en la que se conjugue la necesidad de conocer tanto los contenidos generados desde la larga tradición científica como la de profundizar en los contenidos procedimentales propios del historiador. Una enseñanza de la historia en la que se plantee, por ejemplo, el trabajo directo con fuentes y la necesidad de enfrentarse a las diversas interpretaciones sobre determinados procesos o hechos (CHAPMAN, 2011c). Saber trabajar con interpretaciones en lugar de certezas implica desarrollar un pensamiento crítico sobre las diferentes formas en las que los grupos humanos perciben los ritmos de cambios y permanencias a lo largo del tiempo. No se trata, al fin y al cabo, de que los alumnos de niveles educativos preuniversitarios se formen como historiadores, sino que a través de comprender la historia y pensar sobre esta disciplina, sean capaces de valorar las diferentes argumentaciones o afirmaciones que se utilizan del pasado para describir nuestras sociedades presentes.

No cabe duda de que, desde una óptica cívica, son muchos los beneficios que ofrece una formación histórica que conjugue ambos tipos de contenidos (los de primer y segundo orden) pueden tener para el común de la sociedad y para una adecuada 

study") y Bruce VanSledright y Kimberly Reddy ("Epistemic Beliefs in History: An Exploratory Study Among Prospective Teachers"). En el primero de ellos, centrado en el caso de la enseñanza de la historia en el estado australiano de Nueva Gales del Sur, se plantea justamente el debate que suele producirse entre aquellos que defienden que en los currículos escolares se ha de priorizar la enseñanza de contenidos de primer orden (esto es, fomentar el conocimiento histórico), frente a aquellos otros que abogan por la potenciación de habilidades relacionadas con el pensamiento histórico. Dos posturas que suelen aparecer contrapuestas y en las que, generalmente, suelen vencer aquellos que defienden la enseñanza de contenidos sustantivos o de primer orden. Algo lleva a que, en ocasiones como la descrita, se tengan que buscar "soluciones de compromiso", como es la inclusión de asignaturas no obligatorias en las que se introduce el estudio de la historiografía y se pide a los estudiantes que apliquen sus conocimientos en una investigación histórica original aunando así teoría histórica compleja con la labor del historiador. Unas asignaturas con las que se ha conseguido fomentar la construcción del pensamiento histórico del alumnado, y que muestran claramente la gran función social y educativa de la historia, pero que siguen siendo minoritarias en un currículo en el que la mayor parte de los contenidos impartidos siguen teniendo un claro matiz nacional y basado en los contenidos conceptuales.

Pero el problema no se encuentra tan sólo en los currículos educativos, sino también en la percepción que parte de los docentes (o futuros docentes) de historia tienen sobre la funcionalidad de la enseñanza de la misma motivados por el escaso conocimiento epistemológico de la disciplina que han estudiado y que están dispuestos a enseñar. Como decíamos, esta temática es analizada en el trabajo de VanSledright y Reddy en el que se analizan las creencias epistemológicas sobre el dominio de la disciplina de profesores de historia que recibieron un curso específico sobre epistemología de la disciplina. Pese a que muchos de los alumnos encuestados variaron su consideración sobre el papel y la funcionalidad de la historia, otros alumnos siguieron teniendo una 

disciplina.

No cabe duda de que el reto es tan complejo como necesario de abordar, y buen ejemplo de ello son el más que considerable número de trabajos que han tratado en las dos últimas décadas la cuestión del valor educativo de los conocimientos históricos, qué historia se debe enseñar y de qué manera hay que hacerlo (CARRETERO y VOSS, 2004; CLARK, 2011; PRATS y SANTACANA, 2011; STEARNS, SEIXAS Y WINEBURG, 2000; VANSLEDRIGHT, 2011; WINEBURG, 2001). En este sentido, Peck y Seixas (2008) diferencian entre las tres grandes maneras en la que se suele concebir la educación histórica del alumnado: la primera se centra en la narrativa de la construcción de la nación; la segunda manera tiende al análisis de problemas contemporáneos en un contexto histórico (una forma de enseñar historia más cercano al enfoque de los estudios sociales); y la tercera se centra en comprender la historia como un método, como una manera de investigar desde esta área de conocimiento y, por lo tanto, aprender a pensar y reflexionar con la historia. Esta última sitúa a esta disciplina con un lenguaje y una lógica propia, y que hace uso de esas herramientas para generar nuevos conocimientos (ARTEAGA y CAMARGO, 2013).

Aunque no se trata de tres concepciones de la enseñanza de la historia necesariamente incompatibles, no cabe duda que la tercera de ellas es la que más puede facilitar la formación en el alumnado de pensamiento histórico. Según Seixas y Morton (2012), el pensamiento histórico puede definirse como el proceso creativo que realizan los historiadores para interpretar las fuentes del pasado y generar las narrativas históricas. Para su desarrollo se debe tener en cuenta seis conceptos clave: relevancia histórica; fuentes; cambio y continuidad; causas y consecuencias; perspectiva histórica; y la dimensión ética de la historia. Esta propuesta debe insertarse en el contexto del Centre for the Study of Historical Consciousness de Canadá, dirigido por Peter Seixas. Este centro ha realizado un gran esfuerzo por delimitar la definición de conciencia histórica y pensamiento histórico, y adecuarlos de una forma práctica a la realidad de las aulas a través de 

monografías que han incidido en la definición de ese conocimiento sobre historia. No obstante, esta tendencia también ha estado presente en el continente europeo ya que desde los años 60 en Inglaterra se venía trabajando desde las Universidades de Leeds y Londres en investigaciones y proyectos conducentes a mejorar la comprensión -que no sólo la enseñanza- de la historia conducidos por Peter Lee, Alaric Dickinson y otros (SILVA, 2012). De ahí surgieron proyectos tan interesantes en la década de los 90 como "Concepts of History and Teaching Approaches" (CHATA project) o el "Youth and History" conducente a valorar la conciencia histórica de los jóvenes europeos bajo el auspicio de la European Standing Conferene of Teachers Association (Euroclio), y que ha tenido su correlato en la década del 2000 en Latinoamérica con proyectos como "Jovenes diante da História" (CERRI y AMÉZOLA, 2010).

En el libro colectivo editado por Stearns, Seixas y Wineburg (2000), con aportaciones de autores como Lee, Ashby, Levstik o Wertsch, ya se realizó una amplia reflexión sobre los retos en la enseñanza de la historia en el cambio de siglo y milenio. En esta obra se plantearon los principales temas presentes en la educación histórica a finales de la década de 1990, los cambios necesarios para avanzar en una correcta enseñanza de la historia, o los elementos claves para la investigación sobre la enseñanza y aprendizaje de esta disciplina. En esta línea, en el año 2001 Wineburg publicó Historical Thinking and Other Unnatural Acts cuyas reflexiones han ayudado a sintetizar de manera clara cuáles son la finalidad educativa de la historia, los retos para los estudiantes y profesores en este nuevo enfoque de enseñanza y comprensión histórica, o la confrontación entre la memoria colectiva y el pensamiento histórico. En esta obra se incide en que las habilidades de pensamiento histórico, como el uso de fuentes o la capacidad de plantear problemas históricos en su complejidad no son capacidades naturales que se adquieren con la maduración psicológica, sino que son habilidades que se aprenden y que, por tanto, han de explicitarse en la enseñanza. En la década siguiente, trabajos como los de Barton y Levstik (2004) o el más reciente de VanSledright (2011), han avanzado en la 

donde los alumnos deben desarrollar más complejas habilidades cognitivas para la comprensión y explicación de los fenómenos históricos.

Por tanto, la enseñanza de la historia exige un esfuerzo que va más allá de la memorización de un producto dado. Exige la comprensión de la naturaleza propia de la disciplina y de la actitud propia del investigador que reflexiona sobre el pasado. Es precisamente esta forma de entender la historia la que permite movilizar, tanto en el alumnado como en los investigadores, una serie de habilidades que, según Sáiz (2013), deben actuar en cuatro grandes ámbitos. En primer lugar, el planteamiento de problemas históricos. Se trata en este caso de presentar el significado del pasado y su conocimiento como una realidad no estática, sino en construcción continua a partir de interrogantes de investigación. En segundo lugar, el análisis y obtención de evidencias a partir de fuentes y pruebas históricas, entendido como un pensamiento complejo y creativo, de examen crítico de testimonios del pasado. Esta noción es clave, pues involucra la cuestión de cómo conocer el pasado, y de reconocimiento de la historia como una disciplina que permite estudiarlo a partir de evidencias (ARTEAGA y CAMARGO, 2013). Se trata de ir más allá de la simple decodificación de información textual o icónica. En tercer lugar, el desarrollo de una conciencia histórica, entendida como la capacidad para interrelacionar fenómenos del pasado y del presente. En cuarto lugar, la construcción o representación narrativa del pasado histórico, como la capacidad para comunicar verbalmente, de forma oral o escrita, relatos o explicaciones argumentadas sobre el pasado construidas de forma racional utilizando relaciones causales basadas en pruebas o evidencias históricas.

Tras este visión panorámica y cronológica de los esfuerzos realizados por dotar a la enseñanza de la historia de una metodología que ayuda a desarrollar en el alumnado un espíritu crítico y un conocimiento de la disciplina que permite superar las críticas del conocimiento histórico como producto de construcciones subjetivas de los historiadores, podemos afirmar que existe una coincidencia clara que debe unir tanto la historia académica como la enseñanza de dicha disciplina. Ya sea bien como conceptos clave bien 

sociedades; la argumentación histórica y la correcta articulación del discurso a través del uso crítico de fuentes -lo que implica necesariamente el desarrollo de la causalidad-; y la conexión del pasado con el presente -conciencia histórica-. Pasemos, pues, a analizar con un poco más de detalle el significado de estos tres pilares y los avances llevados a cabo en cada uno de ellos.

El concepto de relevancia histórica implica la capacidad de interrogarnos sobre qué y quién del pasado vale la pena ser recordado y estudiado. La importancia o relevancia del pasado que se debe enseñar y aprender depende del enfoque o la perspectiva desde la que se muestre la disciplina histórica, la duración, el impacto social de ese proceso o acontecimiento histórico y su importancia particular en el contexto donde se enseña. Porque hay que tener muy en cuenta que la relevancia histórica debe estar también vinculada al propio conocimiento escolar concreto, que tiene unas características propias, a veces alejadas de la investigación histórica académica (PLÁ, 2013). Es cierto que, en lo relativo a la selección de lo que resulta relevante como conocimiento escolar, son los propios currículos educativos los que marcan la pauta y, por lo tanto, en muchos casos esa selección acaba estando muy relacionada con decisiones de índole política y no tanto académica. El caso de México, que analiza el profesor Sebastián Plá ("Calidad educativa y didáctica de la historia en los gobiernos neoconservadores en México 2000-2012”), resulta muy interesante en a este respecto: en los últimos años se han hecho muchas mejoras en el currículo escolar, tendentes a incluir en el mismo aspectos relacionados con el fomento del pensamiento histórico entre el alumnado, con lo cual se ha tratado de evitar una historia memorística y enciclopédica para enseñar una historia más formativa y reflexiva. Sin embargo, en lo referente a qué se ha de enseñar (es decir, qué se considera relevante o no), se ha seguido un claro componente ideológico en el que los gobiernos neoconservadores han intentado “europeizar" la historia mexicana, apostando por aumentar el temario dedicado a la historia virreinal a costa de disminuir, hasta casi eliminar del currículo (sólo tiene 

histórico enseñado en las aulas.

En contraste con el ejemplo mexicano, contamos con el caso francés que nos ofrece el profesor Benoît Falaize ("L'enseignement des sujets controversés dans l'école française: les nouveaux fondements de I'histoire scolaire en France?”). En Francia se asiste, desde comienzos de los años setenta, a un auténtica "desacralización de la historia nacional" que huye de la enseñanza de la tradicional historia inmóvil, chauvinista y jalonada de figuras heroicas. Esta tendencia se ha agudizado en las últimas reformas curriculares producidas en las dos últimas décadas con la inclusión de los llamados "sujetos sensibles" (el genocidio armenio, la Sohah, la represión a los movimientos independentistas en las antiguas colonias francesas, la historia de la inmigración...), con los cuales, además de fomentar el debate, la controversia, el constructivismo crítico, supone dar relevancia histórica a una serie de temáticas que, alejadas de la mitificación del relato nacional decimonónico, se encuentran más próximas a un alumnado diverso, propio de una sociedad multicultural como la francesa actual.

Por último, no podemos minusvalorar la importancia de la historiografía en la consideración de qué es relevante como materia de enseñanza histórica. En este sentido, no cabe duda que la historia social ha tenido un papel de primer orden en la inclusión de ciertas temáticas en los programas educativos (MIRALLES, MOLINA y ORTUÑO, 2011). Seixas y Morton (2012) plantean la necesidad de enlazar la vida cotidiana de los hombres y mujeres del pasado con los grandes procesos históricos, algo que, obsta decir, se encuentra entre los objetivos fundamentales de la historia social (GÓMEZ y MIRALLES, 2013). Los trabajos en este dossier de Hernández y Rodríguez ("La Historia Social de la familia en España y su repercusión en la Didáctica de las Ciencias Sociales”), y Sánchez y Miralles ("La historia de las mujeres en las aulas de Educación Secundaria y Bachillerato: algunas propuestas para fomentar el pensamiento histórico sin desigualdades de género"), son buen ejemplo de ello. Como bien señalan los primeros, la historia social, configurada por una amplia serie de problemas históricos concretos (movilidad y cambio 

currículos españoles. Es cierto que, como señalan Miralles y Sánchez, la historia de las mujeres no es uno de esos temas: en el ámbito educativo español y, en concreto, en las etapas de Educación Secundaria Obligatoria y Bachillerato la presencia de las mujeres en los materiales curriculares es todavía escasa. No obstante, también es cierto que una de las razones de que actualmente se plantee en la escuela la necesidad de que el alumnado adquiera la destreza de pensar históricamente sin atisbo de marginación de género, es sin lugar a dudas, el aumento de estudios de género que se ha producido en la historiografía española en los últimos tiempos.

En cuanto al segundo de los grandes elementos constitutivos del pensamiento histórico, el trabajo con fuentes primarias, se trata de uno de los temas que más pone en relación al pensamiento histórico con un proceso metodológico (ÉTHIER, DEMERS y LEFRANÇOIS, 2010). En la construcción del objeto de estudio (el pasado) por los historiadores existe cierto grado de subjetividad, consecuencia del contexto social, cultural, económico o político. Por ello es tan importante insistir en que las huellas, fuentes y evidencias del pasado son muy útiles para la reconstrucción histórica. Pero éstas deben ser evaluadas siguiendo un método riguroso que permita validar las narrativas históricas. En España y en el ámbito latinoamericano el uso de las fuentes es uno de los elementos de pensamiento histórico que más se ha planteado. En aquellos trabajos que reflexionan sobre la finalidad de la enseñanza de la historia, normalmente se acaba haciendo mención a la necesidad de que el alumno aprenda a simular la labor del historiador y con ello que se familiarice a formular hipótesis, aprender a clasificar y analizar fuentes históricas, el aprendizaje de la causalidad y a iniciarse en la explicación histórica (HENRÍQUEZ, 2011; HENRÍQUEZ y RUIZ, 2012; PRATS, 2010; PRATS y SANTACANA, 2011; PRIETO, GÓMEZ y MIRALLES, 2012). Por su parte, en Estados Unidos, son muchos los autores que defienden que se debe trabajar con fuentes desde las etapas educativas iniciales (LESH, 2011; VANSLEDRIGHT 2002 y 2011; WINEBURG, SMITH y BREAKSTONE, 2012). 
El uso de fuentes también está relacionado con la transmisión del conocimiento histórico de una forma compleja, pues su buen uso implica también la aplicación de una correcta argumentación, la capacidad de plantear causas y consecuencias, así como la comprensión de los cambios y permanencias de un proceso histórico desde una perspectiva multifactorial (MORA y ORTIZ, 2013). En este sentido hay que destacar, como ya hemos indicado, los trabajos británicos a través del proyecto CHATA (LEE y ASHBY, 1987 y 2000; LEE et al., 1996 y 2004; LEE 2005; LEE, ASHBY y DICKINSON, 2004). Si bien este proyecto se llevó a cabo en la década de 1990, ya desde la década anterior se venían desarrollando proyectos previos como el "School History Project" en Leeds o el "Cambridge History Project" que terminaron dando forma a CHATA. Todos estos proyectos se basaron en el análisis de las habilidades históricas de los estudiantes mediante el uso de fuentes y ejercicios de empatía histórica. Más recientemente los trabajos de este grupo se han orientado a las argumentaciones del alumnado en su explicación de los procesos históricos, combinando el manejo y crítica de fuentes por parte de este alumnado con las diferentes formas de plantear la causalidad de los hechos históricos. Las experiencias de aprendizaje a partir de la empatía histórica, también desarrolladas en otros países anglosajones como Canadá (BRIANT y CLARK, 2006), acabaron consolidándola como una competencia propia de la capacidad de pensar históricamente (LÉVESQUE, 2008, 2011). Por su parte, y volviendo al ámbito británico, los trabajos de Chapman (2009, 2011a y 2011b) y el proyecto "Constructing History" 1119(COOPER y CHAPMAN, 2009) han profundizado en las argumentaciones del alumnado, y en la formación del pensamiento histórico a través del razonamiento en el uso de fuentes y en una estructura coherente del discurso. Una línea temática que también se ha seguido en otros puntos de Europa, como es el caso de Holanda a través de Van Drie y Van Boxtel (2008). En el caso norteamericano también son abundantes los trabajos que han incidido en las habilidades de pensar, leer, escribir y argumentar históricamente, y en la alfabetización histórica (MONTESANO, 2010; WINEBURG, MARTIN y MONTESANO, 2013). Estos trabajos han mostrado diversos niveles de comprensión y explicación histórica del alumnado, desde el más sencillo, que juzga a los actores históricos según sistemas de valores actuales o presentistas, hasta el más desarrollado que valora y juzga 
Por último, el tercer gran elemento del pensamiento histórico, el desarrollo de una conciencia histórica, esa capacidad para interrelacionar fenómenos del pasado y del presente, supone desarrollar la noción de que todo presente tiene su origen en el pasado; la certeza de que las sociedades no son estáticas, sino que están sujetas a transformaciones; que estas transformaciones constituyen las condiciones del presente; y que cada individuo tiene un papel en ese proceso de transformación social, y por lo tanto el pasado forma parte del propio individuo (SÁIZ, 2013). Sin duda la aportación de la historiografía alemana, como ya hemos apuntado, está detrás de este concepto alemana que ha influenciado mucho, a su vez, la producción científica realizada en este campo en el ámbito portugués (Barca, 2000 y 2011) y brasileño (Schmidt y Garcia, 2005). Pero también hay que destacar el ya citado Centre for the Study of Historical Consciousness, principalmente en la relación entre el concepto de conciencia histórica y pensamiento histórico.

Aunque con cierta frecuencia se suele relacionar la memoria colectiva o memoria histórica con la conciencia histórica, esta última tiene un significado más amplio y más complejo. Si bien la memoria histórica es un constructo social del pasado, basado en la interacción entre los individuos con los grupos a los cuales se adhieren (ÉTHIER, DEMERS y LEFRANÇOIS, 2010), la conciencia histórica opera parcialmente de forma reflexiva y consciente, y está relacionada con la interpretación del tiempo por parte de un sujeto en base a acontecimientos anteriores. Es un procedimiento mental que crea significado de la experiencia del tiempo, mediante la interpretación del pasado, con el fin de entender el presente y sentar expectativas de futuro (SEIXAS, 2004). En este sentido se sitúa el trabajo que nos ofrece Marcelo Fronza ("As concepções de verdade histórica e intersubjetividade no conhecimento histórico de jovens estudantes do Ensino Médio"), en el que analiza, a través de las narrativas históricas que construye el alumnado de educación secundaria, cómo comprenden la relación entre verdad histórica e intersubjetividad (esa verdad histórica sin puntos de intersección de los procesos 
acerca de las ideas de conciencia histórica en el ámbito educativo así como una tradición historiográfica que sustenta ese "hacer diferente" en la enseñanza de la disciplina histórica que supone el pensamiento histórico.

No quisiéramos terminar sin hacer una pequeña reflexión sobre la pertinencia de este monográfico sobre la enseñanza de la historia y el pensamiento histórico. Hemos pretendido mostrar, en primer lugar, hasta qué punto se produce la traslación de conocimientos científicos a la sociedad y por lo tanto el resultado y grado de difusión que los avances en conocimiento histórico están consiguiendo. Y por otro lado, hemos querido defender una disciplina cuyo conocimiento se muestra capaz de resolver interrogantes sociales lo que hace concebir la historia como un saber útil y necesario en el mundo actual. Este es un monográfico que, en nuestra opinión, presenta un alto grado de interés para todos aquellos estudiantes de historia que deseen dedicarse en un futuro a la enseñanza y para aquellos formadores de maestros que actualmente hacen de la enseñanza de la historia su profesión. Según se indica en el manifiesto surgido el año 2001 por el foro internacional Historia a Debate (http://www.h-debate.com/), la historia es "una ciencia con sujeto humano que descubre el pasado conforme lo construye." Es precisamente en este postulado en el que se asienta la contribución del presente dossier a la disciplina histórica, ya que haciendo hincapié en cómo facilitar a cada generación la revisión histórica por medio de la enseñanza del pensamiento histórico estamos consiguiendo fortalecer el conocimiento epistemológico de la disciplina al enseñar a tomar en consideración las dos subjetividades que influyen en nuestro proceso de conocimiento sobre la historia, a saber: la existencia de agentes históricos e historiadores. Conseguir transformar la enseñanza de la historia para dirigirla a este fin es la mejor garantía de la rigurosidad de sus resultados. Como afirma el manifiesto de Historia a Debate, "ha llegado la hora de que la historia ponga al día su concepto de ciencia, abandonando el objetivismo ingenuo heredado del positivismo del siglo XIX, sin caer en el radical subjetivismo resucitado por la corriente posmoderna a finales del siglo 
XX". Creemos firmemente que este dossier podrá contribuir al prestigio académico y social de las investigaciones históricas comenzando por la base de nuestra sociedad, que es la formación educativa. De este modo, al formar en un verdadero pensamiento histórico en las aulas conseguiremos por un lado desarrollar las habilidades propias del pensamiento crítico que el método de trabajo del historiador potencia, al tiempo que establecemos las bases para que futuros jóvenes se interesen por la historia y cuenten con el suficiente bagaje conceptual y procedimental que permitan renovar los temas, las preguntas y las respuestas que las investigaciones históricas requieren. En definitiva, devolver al oficio de historiador el entusiasmo por la renovación y por los compromisos historiográficos.

\section{Referencias}

ARTEAGA, Belinda y CAMARGO, Siddharta. Educación histórica. Una propuesta para el desarrollo del pensamiento histórico en los estudiantes de licenciatura en educación preescolar y primaria. In: PRATS, Joaquim et al. (Ed.). Historia e identidades culturales. Braga: Universidad do Minho, 2013, p. 220-233.

ASHBY, Rosalyn; LEE, Peter. Children's concepts of empathy and understanding in history. In: PORTAL, Ch. (Ed.). The history curriculum for teachers. Londres: Falmer, 1987 p. 62-88.

BARCA, Isabel. O pensamento histórico dos jovens. Braga: Universidade do Minho, 2000.

BARCA, Isabel. La evaluación de los aprendizajes en historia. In: MIRALLES, Pedro; MOLINA, Sebastián; SANTISTEBAN, Andoni (Eds.). La evaluación y el proceso de enseñanza y aprendizaje de las ciencias sociales. vol. I, Murcia: AUPDCS, 2011, p. 107-120.

BARTON, Keith. Research on Students. Ideas about history. In: LEVSTIK, Linda y TYSON, Cynthia. (Eds.). Handbook of research in social studies education. New York: Routledge, 2008.

BARTON, Keith y LEVSTIK, Linda. Teaching history for the common good. New Jersey: Lawrence Erlbaum, 2004.

BRYANT, Darren; CLARK, Penney. Historical empathy and Canada People's History. Canadian Journal of Education. v. 29, n. 4, p.1039-1064, 2006. 
CARRETERO, Mario. Comprensión y aprendizaje de la historia. In: PRATS, Joaquín. et al. Enseñanza y aprendizaje de la historia en la educación básica. México D.F: Secretaría de Educación Pública, Gobierno de México, 2011, p. 69-104.

CARRETERO, Mario y VOSS, James (Comp.). Aprender y pensar la historia. Buenos Aires: Amorrortu, 2004.

CARRETERO, Mario y LÓPEZ, César. Estudios cognitivos sobre el conocimiento histórico: aportaciones para la enseñanza y la alfabetización histórica. Enseñanza de las Ciencias Sociales, v. 9, p. 79-83, 2009.

CATAÑO BALSEIRO, Carmen Lucia. Jörn Rüsen y la conciencia histórica. Historia y Sociedad, v. 21, p. 221-243, 2010.

CERRI, Luis Fernando, AMÉZOLA, Gonzalo. de. El estudio empírico de la conciencia histórica en jóvenes de Brasil, Argentia y Uruguay. Didáctica de las Ciencias Experimentales y Sociales. v. 24, p. 3-23, 2010.

CHAPMAN, Arthur. Introduction: constructing history 11-19. In: Cooper, Hilary y Chapman, Arthur (Ed.). Constructing History, 11-19. Londres: Sage, 2009, p. 1-8.

CHAPMAN, Arthur. Understanding Historical Knowing: Evidence and Accounts. In PERIKLEOUS, Lukas, SHEMILT, Denis (eds.). The Future of the past: why history education matters. Nicosia: Association for Historical Dialogue and Research, Kailas Printers, 2011a, p. $169-216$.

CHAPMAN, Arthur. Taking the perspective of the other seriously? understanding historical argument. Educar em Revista, v. 42, p. 95-106, 2011 b.

CHAPMAN, Arthur. Historical interpretations. In Davies, lan (Ed.). Debates in history teaching. Oxon: Routledge, 2011c, p. 96-109.

CLARK, Penney (Ed.). New possibilities for the past: shaping history education in Canada. Vancouver-Toronto: UBC Press, 2011.

COOPER, Hilary; CHAPMAN, Arthur (Eds.). Constructing History, 11-19. Londres: Sage, 2009.

ÉTHIER, Marc-André; DEMERS, Stéphanie Y LEFRANÇOIS, David. Las investigaciones en didáctica sobre el desarrollo del pensamiento histórico en la enseñanza primaria. Una panorámica de la literatura publicada en francés e inglés desde el año 1990. Enseñanza de las Ciencias Sociales. Revista de Investigación, v. 9, p. 61-74, 2010. 
GÓMEZ, Cosme Jesus. y MIRALLES, Pedro. La enseñanza de la historia desde un enfoque social. Clío. History and History Teaching, n. 39, 2013

HENRÍQUEZ, Rodrigo. Aprender a explicar el pasado: el rol de la causalidad y las evidencias históricas en el aprendizaje de la historia de alumnos chilenos. In: LÓPEZ FACAL, R. et al. (Ed.). Pensar históricamente en tiempos de globalización. Santiago de Compostela: USC, 2011, p. 91-98.

HENRÍQUEZ, RodrigoY RUIZ, Marcela. Enseñar historia o alfabetizar históricamente: el aprendizaje del pensamiento histórico a través del uso de evidencias en la escritura de estudiantes chilenos. In: Anais.... Segundo Congreso Interdisciplinario de Investigación en Educación, Centro de Investigación Avanzada en Educación, Universidad de Chile, 23-24 agosto 2012, http://www.ciie2012.cl/download.php?file=sesiones/46.pdf, (3 de junio de 2013).

LEE, Peter. Historical literacy: theory and research. International Journal of Historical Learning, Teaching and Research, v. 5, n.1, p. 29-40, 2005 a.

LEE, Peter. Putting principles intro practice: understanding history. In: DONOVAN, M. Suzanne, BRANSFORD, John (Ed.). How students learn: history in the classroom. Whasington: National Academies Press, 2005b, p. 31-77.

LEE, Peter. Historical literacy and transformative history. In: PERIKLEOUS, L., SHEMILT, D. (Eds.). The Future of the past: why history education matters. Nicosia: Association for Historical Dialogue and Research, 2011, p. 129-168.

LEE, Peter; ASHBY, Rosalyn. Progression in Historical Understanding among Students ages 7-14. In: STEARNS, Peter N., SEIXAS, Peter, WINEBURG, Samuel(Eds.). Knowing, teaching and learning history: national and international perspectives. Nueva YorkLondres: New York University Press, 2000, p. 199-222.

LEE, Peter; ASHBY, Rosalyn; DICKINSON, Alaric. Progression in children's ideas about history: project CHATA. In: HUGHES, Martin. (Ed.). Progression in Learning. Clevedon: Multilingual Matters, 1996, p. 50-81.

LEE, Peter; ASHBY, Rosalyn, DICKINSON, Alaric. Las ideas de los niños sobre la historia. In: CARRETERO, Mario y VOSS, James (comp.). Aprender y pensar la historia. Buenos Aires: Amorrortu, 2004, p. 217-248.

LEE, Peter; SHEMILT, Denis. 'I just wish we could go back in the past and find out what really happened'. Progression in understanding about historical accounts. Teaching History, v. 117, p. 25-31, 2004. 
LESH, Bruce A. Why won't you just tell us the answer? Teaching historical thinking in grades 7-12. Portland: Stenhouse, 2011.

LÉVESQUE, Stéphane. Thinking historically: educating students for the 21th century. Toronto: University of Toronto Press, 2008.

LEVESQUE, Stéphane. Le pensée historique: pour le développement de le litératie critique en histoire. Canadian Issues / Thèmes canadiens, Éte/summer, 2011, p. 13-16.

LOPEZ FACAL, Ramon et al. (Eds.) Pensar históricamente en tiempos de globalización. Santiago de Compostela: USC, 2011.

MEGILL, Allan. 'Jörn Rüsen's Theory of historiography'. History and Theory, v. 33, n. 1, 1994, p. 39-60.

MILLAN, Jesús. Presentación. El contexto de la historia social crítica en la Alemania contemporánea. In: KOCKA, Jürgen. Historia social y conciencia histórica. Madrid: Marcial Pons, 2002, p. 11-42.

MIRALLES, Pedro; MOLINA, Sebastián y ORTUÑO, Jorge. La importancia de la historiografía en la enseñanza de la historia. Granada: GEU, 2011.

MOLINA, Sebastián; RODRÍGUEZ, RaimundoA. Y SÁNCHEZ, Raquel. Investigar, enseñar y divulgar la historia: la experiencia del Seminario y grupo de investigación familia y élite de poder de la Universidad de Murcia. Clío. History and History Teaching, n. 39, 2013.

MONTE-SANO, Chauncey. Disciplinary literacy in history: an exploration of the historical nature of adolescents' writing. The Journal of the Learning Sciences, v. 19, n.4, p. 539-568, 2010.

MORA, Gerardo Y Paz Rosa Ortiz. La enseñanza del "tiempo histórico": problemas y propuestas didácticas. História \& Ensino, v. 19, n.1, p. 7-25, 2013.

PECK, Carla; SEIXAS, Peter. Benchmarks of Historical thinking: first steps. Canadian Journal of Education, v. 31, n. 4, p. 1015-1038, 2008.

PLA, Sebastián. La ilusión científica de la didáctica de la historia. Provocaciones teóricas sobre el conocimiento histórico escolar. In: Anais.... Cuarto Encuentro Nacional de Docencia, Difusión y Enseñanza de la Historia. Santiago de Querétaro: Universidad Autónoma de Querétaro, 2013.

PRATS, Joaquín. En defensa de la historia como materia educativa.Tejuelo, v. 9, p. 8-18, 2010. 
PRATS, Joaquín y SANTACANA, Joan. ¿Por qué y para qué enseñar historia? In Prats, Joaquín(Coord.). Didáctica de la geografía y la historia. Barcelona: Graó, 2011, p. 13-29.

PRIETO, José A.; GÓMEZ, Cosme J. y MIRALLES, Pedro. El uso de fuentes primarias en el aula y el desarrollo del pensamiento histórico y social: una experiencia en Bachillerato. Clío. History and History Teaching, n. 39, 2013

RÜSEN, Jörn. History: narration, interpretation, orientation. Nueva York: Berghahn, 2005.

SÁIZ, Jorge. Alfabetización histórica y competencias básicas en libros de texto de historia y en aprendizaje de los estudiantes. Didáctica de las Ciencias Experimentales y Sociales, v. 27, p. 43-66, 2013.

SEIXAS, Peter. A Modest Proposal for Change in Canadian History Education. In NAKOU, Eirene, BARCA, Isabel. (Ed.). Contemporany public debates over history education. Charlotte NC: Information Age Publishing, 2010, p. 11-26

SEIXAS, Peter. Assesment of Historical Thinking. In CLARK, P. (Ed.). New possibilities for the past: shaping history education in Canada. Vancouver-Toronto: UBC Press, 2011, p. 139153.

SEIXAS, Peter (Ed.). Theorizing historical consciousness. Toronto: Toronto University Press, 2004

SEIXAS, Peter y MORTON, Tom. The big six historical thinking concepts. Toronto: Nelson, 2012.

SCHMIDT, Maria Auxiliadora Moreira dos Santos y GARCÍA, Tânia Maria Braga. A formação da consciência histórica de alunos e professores e o cotidiano em aulas de História. Cad. Cedes, Campinas, v. 25, n. 67, p. 297-308, set./dez. 2005. Disponible en <http://www.cedes.unicamp.br/caderno/cad/cad67.htm>

SILVA, Cristiani Bereta da. O ensino de História: álgunas refexôes do Reino Unido: entrevista com Peter J. Lee". Tempo e Argumento. v. 4, n. 2, p. 216-250, 2012.

STEARNS, Peter N.; SEIXAS, Peter y WINEBURG, Samuel(Ed.). Knowing, Teaching and learning history: national and international perspectives. Nueva York-Londres: New York University Press, 2000.

STONE, Lawrence. History and post-modernism. Past and Present, n.131, p. 207-218, 1991. 
VAN DRIE, Jan y VAN BOXTEL, Carla. Historical reasoning: towards a framework for analyzing student's reasoning about the past. Educational Psychology Review. v. 20, p. 87110, 2008.

VANSLEDRIGHT, Bruce A. Insearch of America's pst. Learning to read history in elementary school. New York: Teacher College Press, 2002.

VANSLEDRIGHT, Bruce A. The challenge of rethinking history education: on practice, theories, and policy. New York: Routledge, 2011.

VANSLEDRIGHT, BruceA. Assessing historical thinking and understanding: Innovate designs for new standards. New York: Routledge, 2014.

VATTIMO, Gianni. La sociedad transparente. Milán: editorial Paidós, 1989.

WINEBURG, Samuel. Historical thinking and other unnaturals acts: charting the future of teaching the past. Philadelphia: Temple University Press, 2001.

WINEBURG, Samuel; MARTIN, Daisy y MONTE-SANO, Chauncey. Reading like a historian: teaching literacy in middle \& high school history classrooms. Nueva York: Teachers C, 2013.

WINEBURG, Samuel; SMITH, Mark y BREAKSTONE, Joel. (2012). New directions in assessment using library congress sources to assess historical understanding. Social Education, v. 76, n. 6, p. 288-291, 2012. 
Recebido em 01/12/2013 Aprovado em 10/03/2014

Universidade do Estado de Santa Catarina - UDESC

Programa de Pós-Graduação em História - PPGH

Revista Tempo e Argumento

Volume 06 - Número 11 - Ano 2014

tempoeargumento@gmail.com 